TITLE:

\title{
Elastic inhomogeneity and acoustic phonons in Pd-, Pt-, and Zr-based metallic glasses
}

\section{AUTHOR(S):}

Ichitsubo, T.; Itaka, W.; Matsubara, E.; Kato, H.; Biwa, S.; Hosokawa, S.; Matsuda, K.; ... Yokoyama, Y.; Uchiyama, H.; Baron, A. Q. R.

\section{CITATION:}

Ichitsubo, T.... [et al]. Elastic inhomogeneity and acoustic phonons in Pd-, Pt-, and Zrbased metallic glasses. Physical Review B 2010, 81(17): 172201.

\section{ISSUE DATE:}

2010-05

URL:

http://hdl.handle.net/2433/130710

RIGHT:

(C) 2010 The American Physical Society 


\title{
Elastic inhomogeneity and acoustic phonons in Pd-, Pt-, and Zr-based metallic glasses
}

\author{
T. Ichitsubo, ${ }^{1, *}$ W. Itaka, ${ }^{1}$ E. Matsubara, ${ }^{1}$ H. Kato, ${ }^{2}$ S. Biwa, ${ }^{3}$ S. Hosokawa, ${ }^{4}$ K. Matsuda,${ }^{5}$ J. Saida,${ }^{6}$ O. Haruyama, \\ Y. Yokoyama, ${ }^{2}$ H. Uchiyama, ${ }^{8}$ and A. Q. R. Baron ${ }^{8,9}$ \\ ${ }^{1}$ Department of Materials Science and Engineering, Kyoto University, Kyoto 606-8501, Japan \\ ${ }^{2}$ Institute for Materials Research, Tohoku University, Sendai 980-8577, Japan \\ ${ }^{3}$ Department of Micro Engineering, Kyoto University, Kyoto 606-8501, Japan \\ ${ }^{4}$ Center for Materials Research Using Third-Generation Synchrotron Radiation Facilities, Hiroshima Institute of Technology, \\ Hiroshima 731-5193, Japan \\ ${ }^{5}$ Department of Physics, Graduate School of Science, Kyoto University, Kyoto 606-8502, Japan \\ ${ }^{6}$ Center for Interdisciplinary Research, Tohoku University, Sendai 980-8578, Japan \\ ${ }^{7}$ Department of Physics, Faculty of Science and Technology, Science University of Tokyo, Noda 278-8510, Japan \\ ${ }^{8}$ SPring-8/JASRI, 1-1-1 Kouto, Sayo-cho, Hyogo-ken 679-5198, Japan \\ ${ }^{9}$ SPring-8/RIKEN, 1-1-1 Kouto, Sayo-cho, Hyogo-ken 679-5148, Japan
}

(Received 24 September 2009; revised manuscript received 22 January 2010; published 3 May 2010)

\begin{abstract}
We have investigated acoustic-phonon behavior of Pd-, Pt-, and Zr-based metallic glasses with the combination of inelastic x-ray scattering and ultrasonic (US) measurements. In the dispersion of the longitudinal acoustic modes at small wave number $Q$, the phase velocity of phonon tends to exceed the US velocity with a wavelength of macroscopic scale for Pd- and Pt-based glasses, whereas the former goes close to the latter for Zr-based glasses. Furthermore, in all the cases, the mean free path $L$ of phonon apparently increases steeply at a certain $Q$ value. These phenomena on the acoustic phonon behavior can be qualitatively explained by the elastic-wave-scattering theory with a simple two-phase model.
\end{abstract}

DOI: 10.1103/PhysRevB.81.172201

Disordered materials, liquids and glasses, exhibit complex relaxation processes. ${ }^{1}$ The correlation between these dynamic processes and glass structure is one of the main issues in glass science. One focus has been the tendency of the acoustic mode to disperse faster than expected from measurements of the ultrasonic (US) sound velocity. This "positive dispersion" is frequently observed in many disordered systems. ${ }^{2}$ For liquids, the positive dispersion may be explained within the framework of the mode coupling theory, ${ }^{3}$ in which a fast-rattling process, being distinct from $\alpha$ and $\beta$ (Johari-Goldstein) relaxations, ${ }^{4,5}$ (see for details Ref. 6), is supposed to be present. However, the positive dispersion is also observed in a glassy state, e.g., a silica glass, ${ }^{7-9}$ and the phenomenon has been attributed to the residual fast process in the glass. ${ }^{2,3}$ As was shown by Buchenau, ${ }^{10}$ elastic moduli of glasses are frequency dependent at sufficiently high temperatures where $\alpha$ or $\beta$ relaxation can take place. Hence, the fast process is one candidate for causing the positive dispersion in relatively low-density glassy materials.

Recently, we reported deterioration of the structure of a glass near the glass transition temperature due to the combined action of external megahertz frequency vibrations and $\beta$ relaxation. ${ }^{11,12}$ The partially crystallized microstructure of a Pd-based metallic glass under submegahertz vibration strongly indicates that the atomic motions associated with the Johari-Goldstein $\beta$ relaxation are spatially localized. ${ }^{13,14}$ Based on our experimental results on a transmission electron micrograph, we proposed a microstructural model of metallic glasses that consists of strongly bonded regions surrounded by weakly bonded regions. ${ }^{13}$ To confirm the validity of the model, we have performed inelastic x-ray scattering and ultrasonic measurements, ${ }^{15}$ and also have measured the elastic moduli at low temperatures to freeze out any processes residual in the glass. ${ }^{16}$ In those works, we have experimentally shown that the sound velocity of nanometer wavelength
PACS number(s): 63.50.Lm, 61.20.Lc, 62.40.+i

acoustic phonons exceeds the ultrasound velocity of millimeter wavelength (in macroscopic scale) in the Pd-based metallic glass completely frozen far below the glass transition temperature. Here, we have investigated the acoustic-phonon properties of several Pd-, Pt-, and Zr-based metallic glasses by combining results from inelastic $\mathrm{x}$-ray scattering (IXS) and US techniques. Finally, with the aid of the Yang-Mal's (YM) elastic-wave-scattering theory, ${ }^{17}$ we have suggested that there exists intrinsic elastic inhomogeneity with a certain correlation length in these metallic glasses.

The longitudinal elastic constant $c_{\mathrm{L}}$ and also shear (transverse) constant $c_{\mathrm{T}}$ of a rectangular parallelepiped sample cut out of each ingot were determined using the resonant ultrasound spectroscopy (RUS) technique, ${ }^{18,19}$ to obtain the longitudinal (and also transverse) US velocity in a macroscopic scale, $v_{\mathrm{L}}^{\mathrm{US}}=\sqrt{c_{\mathrm{L}} / \rho}$ (and also $v_{\mathrm{T}}^{\mathrm{US}}=\sqrt{c_{\mathrm{T}} / \rho}$ ). The density, elastic constants, and sound velocity of the samples used in this work are listed in Table I. On the other, each sample for IXS was cut from the same ingot prepared for RUS and was mechanically polished into a thin plate with a thickness of about 50-90 $\mu \mathrm{m}$ appropriate for transmission of X-ray measurements. The IXS measurement was performed at room temperature at the high-resolution IXS beam line, BL35XU of SPring-8 in Japan; ${ }^{20}$ the details in the IXS measurement were described in Ref. 15. Figure 1 shows part of the IXS spectrum at each $Q$ value. The data presented have been corrected for backgrounds by subtracting the empty-vessel response (including the $\mathrm{Si}$ window), after correction for sample attenuation. To obtain the excitation energy at each $Q$ value, the present IXS spectra were fitted to the damped harmonic oscillator (DHO) function, ${ }^{21,22}$

$$
\frac{S(Q, \omega)}{S(Q)}=A_{0} \frac{\Gamma_{0}}{\Gamma_{0}^{2}+\omega^{2}}+A_{Q} \frac{\Omega_{Q}^{2} \Gamma_{Q}}{\left(\omega^{2}-\Omega_{Q}^{2}\right)^{2}+\omega^{2} \Gamma_{Q}^{2}},
$$


TABLE I. The density, elastic constants, and sound velocity of the six bulk metallic glasses measured by RUS.

\begin{tabular}{lcccccc}
\hline \hline Glass sample (abbreviation) & $\begin{array}{c}\mathrm{Pd}_{42.5} \mathrm{Ni}_{7.5} \mathrm{Cu}_{30} \mathrm{P}_{20} \\
(\mathrm{PNCP})\end{array}$ & $\begin{array}{c}\mathrm{Pd}_{46} \mathrm{Cu}_{35.5} \mathrm{P}_{18.5} \\
(\mathrm{PdCP})\end{array}$ & $\begin{array}{c}\mathrm{Pt}_{60} \mathrm{Ni}_{15} \mathrm{P}_{25} \\
(\mathrm{PtNP})\end{array}$ & $\begin{array}{c}\mathrm{Zr}_{50} \mathrm{Cu}_{40} \mathrm{Al}_{10} \\
(\mathrm{ZCA})\end{array}$ & $\begin{array}{c}\mathrm{Zr}_{65} \mathrm{Al}_{7.5} \mathrm{Ni}_{10} \mathrm{Cu}_{17.5} \\
(\mathrm{ZANC})\end{array}$ & $\begin{array}{c}\mathrm{Zr}_{65} \mathrm{Al}_{7.5} \mathrm{Ni}_{10} \mathrm{Pd}_{17.5} \\
(\mathrm{ZANP})\end{array}$ \\
\hline Density, $\rho\left(\mathrm{kg} / \mathrm{m}^{3}\right)$ & 9237 & 9485 & 15270 & 6837 & 6430 & 7145 \\
$c_{\mathrm{L}}(\mathrm{GPa})$ & 209.1 & 208.3 & 257.8 & 158.3 & 144.2 & 165.3 \\
$c_{\mathrm{T}}(\mathrm{GPa})$ & 34.20 & 33.57 & 34.39 & 33.91 & 28.29 & 29.18 \\
$v_{\mathrm{L}}^{\mathrm{US}}(\mathrm{m} / \mathrm{s})$ & 4758 & 4686 & 4109 & 4812 & 4736 & 4810 \\
\hline \hline
\end{tabular}

where $A_{0}$ and $A_{Q}$ are the relative intensities, $\Gamma_{0}$ and $\Gamma_{Q}$ are associated with the sound attenuation, $\Omega_{Q}$ is the characteristic acoustic frequency, and in practice, we included the detailed-balance term and convolved in the instrumental resolution function.

Figure 2(a) shows the phonon-dispersion relation for each glass (lower panel) and the phase velocity of longitudinal phonon, $v_{\mathrm{r}} \equiv v_{\mathrm{L}}^{\mathrm{IXS}} / v_{\mathrm{L}}^{\mathrm{US}}=\Omega_{Q} / Q v_{\mathrm{L}}^{\mathrm{US}}$, normalized by the ultrasound speed, i.e., "relative sound velocity" (upper panel). Although the US velocity in homogeneous solids is usually the fastest at the long-wavelength limit, $v_{\mathrm{r}}$ exceeds 1 for the Pt- and Pd-based metallic glasses (PNCP, PdCP, and PtNP) in the low- $Q$ region. In contrast, for the $\mathrm{Zr}$-based metallic glasses (ZANC, ZANP, and ZCA), the relative velocity tends to approach 1 at low- $Q$ values.

Furthermore, we can evaluate the phonon propagation length (mean free path) from the IXS results; this is taken as $L(Q) \equiv \Omega_{Q} / Q \Gamma_{Q}$. Figure 2(b) shows the $\lambda$ dependence of $L$ for the six metallic glasses. For the sake of intuitive understanding, $L$ is plotted as a function of the wavelength $\lambda$ ( $=2 \pi / Q)$ of phonon. In all the cases, we can see the tendency that $L$ increases with increase in $\lambda$ and changes steeply at a certain $\lambda$ value (defined as $\lambda_{c}$ ). Here we do not intend to define the values of $\lambda_{c}$ and the index of power law, because some of the IXS data are scattering. It may be a characteristic feature that the values of Pd- and Pt-based glasses appear to be larger than those of the $\mathrm{Zr}$-based glasses.
As was discussed by Masciovecchio et al. ${ }^{23}$ the fact that the phonon is well defined at $\lambda>\lambda_{c}$ may imply that there exists such an elastic inhomogeneity with a certain correlation length of the elastic fluctuation, $\xi$, which may be comparable to $\lambda_{c}\left(=2 \pi / Q_{c}\right)$. However, the argument on the correlation length of vitreous silica is under debate, ${ }^{24}$ and to date, we have no theoretical evidence or support for the prediction that the correlation length (domain size) can be evaluated from the $\lambda_{c}$ value at which the mean free path changes steeply.

We thus consider the positive dispersion and steep increase in $L$ around the critical wavelength $\lambda_{c}$ with the aid of the elastic-wave-scattering theory established by Yang and Mal theory, ${ }^{17}$ which has been formulated as a substantial extension of the Waterman-Truell theory ${ }^{25}$ and validated by recent numerical simulations. ${ }^{26,27}$ According to the YM theory, the effective wave number $k_{\text {eff }}$ in the inclusion/ matrix/effective medium composite medium is given by

$$
1=\left(1-\frac{2 i n_{\mathrm{s}} f_{0}}{k_{\mathrm{eff}}^{2}}\right)^{2}-\left(\frac{2 i n_{\mathrm{s}} f_{\pi}}{k_{\mathrm{eff}}^{2}}\right)^{2},
$$

where the forward-scattering and backscattering amplitudes now correspond to the composite (inclusion/matrix) scatterer embedded in the effective medium, $n_{\mathrm{s}}=V_{\mathrm{f}} / \pi a^{2}$ is the number density of inclusions [in a two-dimensional (2D) model], and $f_{0}$ and $f_{\pi}$ are the forward-scattering and backscattering am-
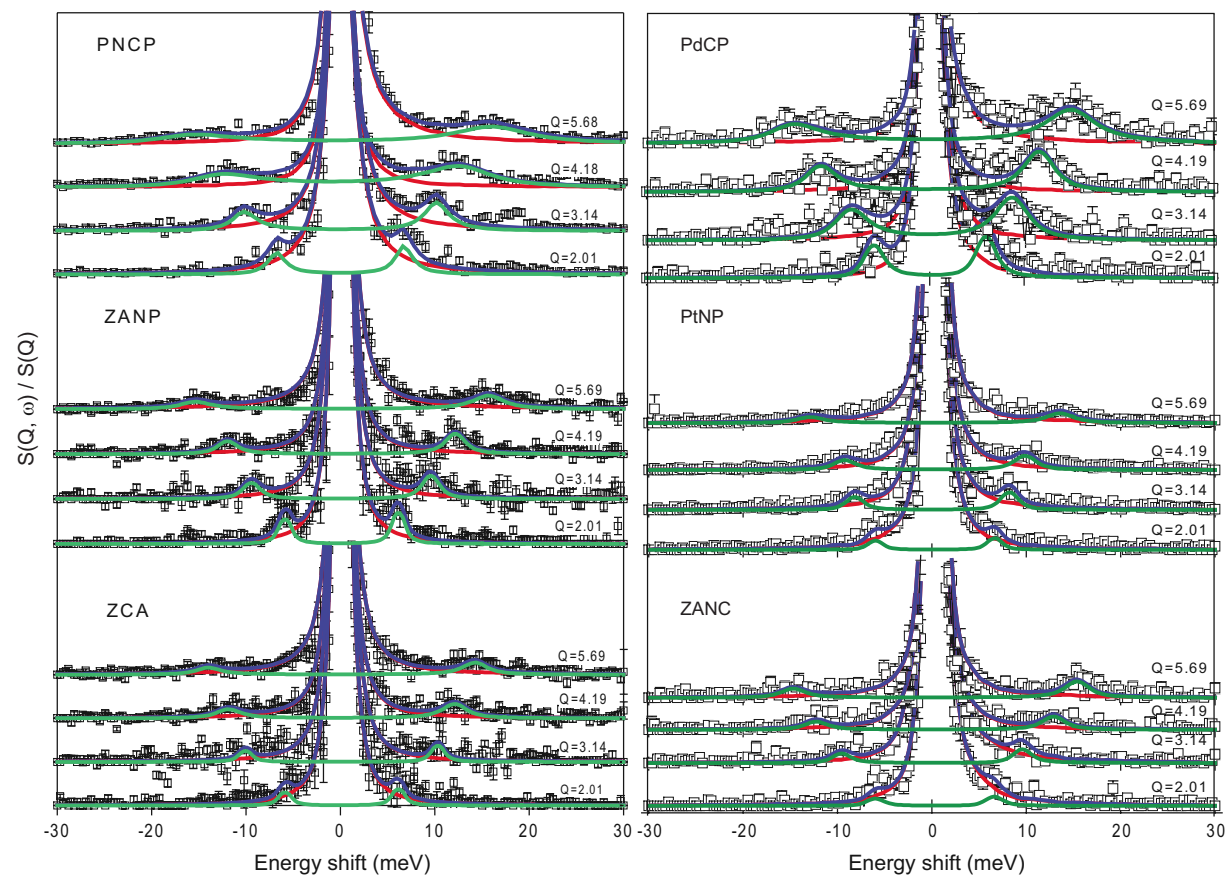

FIG. 1. (Color online) Typical IXS spectra at the some low- $Q$ values obtained for the six glasses. The black circles and three curves indicate raw data and the fitted DHO function Eq. (1) to data, respectively. 
(a)

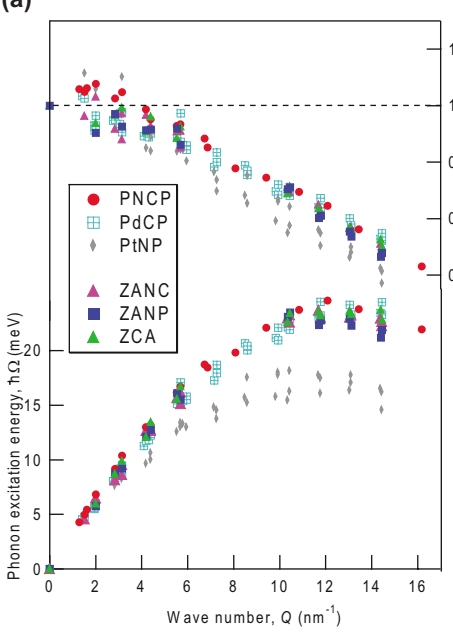

(b)

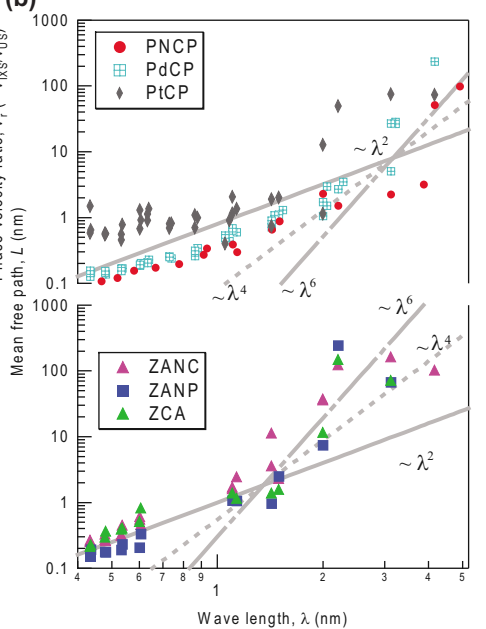

FIG. 2. (Color online) (a) The phonondispersion relation (lower panel) and relative phase velocity to ultrasound, $\Omega_{Q} / Q v_{\mathrm{L}}^{\mathrm{US}}$, (upper panel) of six types of metallic glasses, which were obtained from the IXS spectra. (b) The $\lambda$ dependence of the mean free path $L$ of acoustic phonon obtained for six glasses. plitudes of a single inclusion in the matrix, respectively, for the plane-wave incidence with angular frequency $\omega$. Once the effective wave number $k_{\text {eff }}$ is determined, the phase velocity $V_{\text {eff }}$ and the attenuation coefficient $\alpha_{\text {eff }}$ of the composite are given by the relation $k_{\text {eff }}=\omega / V_{\text {eff }}+i \alpha_{\text {eff }}$. Presently, the propagation length $L$ is defined as $1 / \alpha_{\text {eff }}$.

Here, for the sake of simplicity, we consider a 2D simple model consisting of two regions in Fig. 3(a), in which elastically softer matrix surrounds harder inclusions of volume fraction $V_{\mathrm{f}}$ and deal with only a shear horizontal $(\mathrm{SH})$ wave in a two-dimensional system. In this case, only shear moduli of the two phases are needed for calculations: $G_{\mathrm{M}}=20 \mathrm{GPa}$ (the matrix shear modulus) and $G_{\mathrm{I}}=40 \mathrm{GPa}$ (the inclusion shear modulus). Since actually the inelastic x-ray scattering is dominated by (perhaps mainly sensitive to) longitudinal modes, strictly speaking, we should calculate for the longitudinal mode in three dimensions (3D), and the 2D model cannot express exactly such complicated wave scattering in
3D. However, the only shear wave exists and the longitudinal wave does not in our present 2D model, which means that the shear wave is the fastest. This situation is similar to the longitudinal mode in an actual glass substance. In addition, mode transfer can be neglected in this model, and also in the actual experiment the fastest longitudinal mode was only analyzed and such transferred modes were not detected. From the above viewpoints, it is considered that the actual situation of the longitudinal-wave scattering is partially similar to the shear-wave scattering in the present model. Our trial by the present model calculation is to grasp how the phase velocity and propagation-length behavior change when there is an elastic inhomogeneity with a static correlation length (even in this 2D model).

Figure 3(b) (left panel) shows the effective phase velocity relative to that in the matrix as a function of the inverse of wavelength and (right panel) the propagation length as a function of wavelength for a composite material calculated
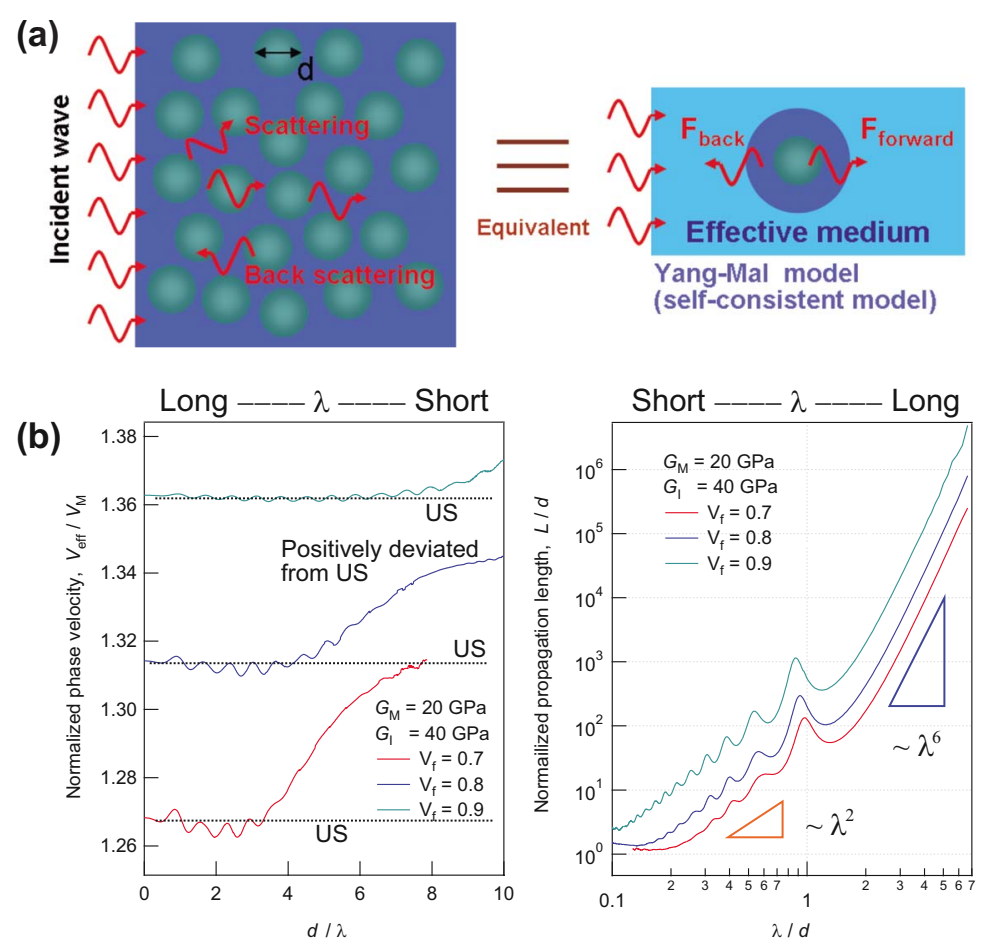

FIG. 3. (Color online) (a) Schematic figure illustrating the two-dimensional heterostructure model of glass, consisting of hard inclusions embedded in a softer matrix. (b) The effective phase velocity relative to that in the matrix as a function of the inverse of wavelength and the propagation length as a function of wavelength for a composite material calculated with the Yang-Mal effective medium self-consistent theory. The volume fraction of inclusion is set at $V_{\mathrm{f}}=0.7,0.8$, and 0.9 . The effective phase velocity is normalized the phase velocity of the matrix $V_{\mathrm{M}}$. $G_{\mathrm{I}}=40 \mathrm{GPa}$ and $G_{\mathrm{M}}=20 \mathrm{GPa}$. This model can be used to express the positive dispersion in the low- $Q$ range. 
by using the YM theory. It should be noted that, in both cases, the effective phase velocity $V_{\text {eff }}$ normalized by the phase velocity of the matrix $V_{\mathrm{M}}$ tends to increase with increase in $d / \lambda$ ( $d$ : diameter of inclusions) that is proportional to the $Q$ value in Fig. 2(a). The $V_{\text {eff }} / V_{M}$ value at $d / \lambda=0$ is the long-wavelength limit, corresponding to the ultrasound value in Fig. 2(b). The point at which $V_{\text {eff }} / V_{\mathrm{M}}$ increases markedly is on the order of the diameter $d$. Thus, the present model can reproduce the characteristic features of the experimental positive dispersion.

When the fraction $V_{\mathrm{f}}$ is larger (i.e., more homogeneous), the degree of increase becomes faint, namely, the positive dispersion tends to depend on the fraction of softer matrix. Regardless of increase or decrease in $V_{\text {eff }} / V_{\mathrm{M}}$, the mean free path $L / d$ (propagation length) tends to increase with increase in $\lambda$ and it obviously changes at $d / \lambda=1$. (The undulation in the low- $\lambda / d$ region is due to the resonance scattering.) Our present model considers only $\mathrm{SH}$ wave (not longitudinal wave) in a simple two-dimensional model; nevertheless, the characteristic feature can be reproduced with this elastic inhomogeneous model. It is worth noting that this drastic change in $L$ around $d / \lambda=1$ can be reproduced by assuming that two phases exist in the substance, whereas the degree of positive dispersion depends on the volume fraction of the two phases. From the present calculations, we can understand the significant feature of the experimental results; that is, all the metallic glasses used in this work do not show the positive dispersion, but all of them exhibit the marked increase in mean free path $L$. Thus, we may conclude that the $\lambda_{c}$ value represents the correlation length $\xi$ of domain (corresponding to the diameter $d$ in this model). By understanding comprehensively the present experimental and theoretical results, it can be affirmed that the degrees of elastic inhomogeneity in PNCP, PdCP, and PtNP glasses are larger than that in ZANC, ZANP, and ZCA glasses.

To conclude, we have investigated systematically the acoustic properties of some Pd-, Pt-, and Zr-based metallic glasses by the combination of IXS and US measurements with the aid of the elastic-wave-scattering theory, to substantiate the elastic inhomogeneity of densely packed metallic glasses. In the cases of $\mathrm{Pd}_{42.5} \mathrm{Ni}_{7.5} \mathrm{Cu}_{30} \mathrm{P}_{20}, \mathrm{Pd}_{46} \mathrm{Cu}_{35.5} \mathrm{P}_{18.5}$, and $\mathrm{Pt}_{60} \mathrm{Ni}_{15} \mathrm{P}_{25}$, the sound velocity of the phonons at low $Q$ exceeds the US velocity, whereas the former goes close to the latter in the cases of $\mathrm{Zr}_{65} \mathrm{Al}_{7.5} \mathrm{Ni}_{10} \mathrm{Pd}_{17.5}$, $\mathrm{Zr}_{65} \mathrm{Al}_{7.5} \mathrm{Ni}_{10} \mathrm{Cu}_{17.5}$, and $\mathrm{Zr}_{50} \mathrm{Cu}_{40} \mathrm{Al}_{10}$. Moreover, the $\lambda(Q)$ dependence of $L \sim \Omega_{Q} / Q \Gamma_{Q}$ representing the mean free path of phonon was also evaluated for the six metallic glasses. The $L$ values of all the glasses used here tend to increase with increase in $\lambda$ and steeply change at a certain value of $\lambda$ $\left(\lambda_{c}\right)$. It is found that the $\lambda_{c}$ values for $\mathrm{Pd}_{42.5} \mathrm{Ni}_{7.5} \mathrm{Cu}_{30} \mathrm{P}_{20}$, $\mathrm{Pd}_{46} \mathrm{Cu}_{35.5} \mathrm{P}_{18.5}$, and $\mathrm{Pt}_{60} \mathrm{Ni}_{15} \mathrm{P}_{25}$ glasses are larger than those for $\mathrm{Zr}$-based metallic glasses. In the light of the elastic-wavescattering theory with the present two-phase model, it is strongly supported that the elastic inhomogeneity with a certain correlation length exists even in dense metallic glasses. As described by Masciovecchio et al. ${ }^{23}$ this critical $\lambda_{c}$ value can reflect the characteristic domain size $\xi$ in glasses, and the present results indicate that $\mathrm{Zr}$-based metallic glasses are more homogeneous than the Pt- and Pd-based metallic glasses.

Although our inhomogeneous model can qualitatively explain our experiments, according to Schirmacher et al., ${ }^{28,29}$ the positive dispersion can be successfully explained even when assuming that the elastic-constant fluctuation has no correlation length. In order to check this, high-angle annular dark field-scanning transmission electron microscopy observation may be a powerful tool for detection of domainlike inhomogeneity in glasses. In addition, as was shown by Rufflé et al. ${ }^{30}$ also in the present metallic glasses the opticlike Boson peak (being less dependent on $Q$ ) may exist in a low- $Q$ region (e.g., around $4 \mathrm{meV}$ ), where we observed the positive dispersion for the present metallic glasses; its existence may be one of the reason of the positive dispersion at low $Q$ 's. However, the correlation between the Boson peak and the propagation of phonon is still unclear and it will be further investigated. At present, our model is surely sufficient to explain the experimental results in terms of both positive dispersion and propagation length of phonon.

The synchrotron radiation experiments were performed at the SPring-8 with the approval of the Japan Synchrotron Radiation Research Institute (JASRI).

\footnotetext{
*tichi@mtl.kyoto-u.ac.jp

${ }^{1}$ P. G. Debenedetti and F. H. Stillinger, Nature (London) 410, 259 (2001).

${ }^{2}$ T. Scopigno et al., Rev. Mod. Phys. 77, 881 (2005).

${ }^{3}$ S. H. Chong, Phys. Rev. E 74, 031205 (2006).

${ }^{4}$ G. P. Johari and M. Goldstein, J. Chem. Phys. 53, 2372 (1970).

${ }^{5}$ G. P. Johari, J. Chem. Phys. 58, 1766 (1973).

${ }^{6}$ P. Lunkenheimer et al., Phys. Rev. E 77, 031506 (2008).

${ }^{7}$ T. Scopigno et al., Phys. Rev. Lett. 92, 025503 (2004).

${ }^{8}$ B. Ruzicka et al., Phys. Rev. B 69, 100201(R) (2004).

${ }^{9}$ T. Scopigno et al., Physica B 318, 341 (2002).

${ }^{10}$ U. Buchenau and A. Wischnewski, Phys. Rev. B 70, 092201 (2004).

${ }^{11}$ T. Ichitsubo et al., Acta Mater. 52, 423 (2004).

${ }^{12}$ T. Ichitsubo et al., Scr. Mater. 49, 267 (2003).

${ }^{13}$ T. Ichitsubo et al., Phys. Rev. Lett. 95, 245501 (2005).

${ }^{14}$ T. Ichitsubo et al., J. Chem. Phys. 125, 154502 (2006).
}

${ }^{15}$ T. Ichitsubo et al., Phys. Rev. B 76, 140201(R) (2007).

${ }^{16}$ T. Ichitsubo et al., Phys. Rev. B 78, 052202 (2008).

${ }^{17}$ R. B. Yang and A. K. Mal, J. Mech. Phys. Solids 42, 1945 (1994).

${ }^{18}$ H. H. Demarest, Jr., J. Acoust. Soc. Am. 49, 768 (1971).

${ }^{19}$ I. Ohno, J. Phys. Earth 24, 355 (1976).

${ }^{20}$ A. Q. R. Baron et al., J. Phys. Chem. Solids 61, 461 (2000).

${ }^{21}$ B. Fåk and B. Dorner, Physica B 234, 1107 (1997).

${ }^{22}$ T. Scopigno et al., Phys. Rev. Lett. 96, 135501 (2006).

${ }^{23}$ C. Masciovecchio et al., Phys. Rev. Lett. 97, 035501 (2006).

${ }^{24}$ A. Devos et al., Phys. Rev. B 77, 100201(R) (2008).

${ }^{25}$ P. C. Waterman and R. Truell, J. Math. Phys. 2, 512 (1961).

${ }^{26}$ S. Biwa et al., Int. J. Solids Struct. 41, 435 (2004).

${ }^{27}$ S. Biwa et al., Mech. Mater. 39, 1 (2007).

${ }^{28}$ W. Schirmacher, Europhys. Lett. 73, 892 (2006).

${ }^{29}$ W. Schirmacher et al., Phys. Rev. Lett. 98, 025501 (2007).

${ }^{30}$ B. Rufflé et al., Phys. Rev. Lett. 96, 045502 (2006). 